\title{
Design Guidelines for Anisotropic Conductive Adhesive Assemblies in Microelectronics Packaging
}

\author{
Melida Chin ${ }^{1}$ \\ S. Jack $\mathrm{Hu}^{2}$ \\ Professor \\ e-mail: jackhu@umich.edu
}

\author{
James R. Barber \\ Department of Mechanical Engineering, \\ The University of Michigan, \\ 2250 G. G. Brown, \\ 2350 Hayward Street, \\ Ann Arbor, Ml 48109-2125
}

Multiple parameters are involved in the design of anisotropic conductive adhesive assemblies, and the overlapping influences that they have on the final electrical contact resistance represent a difficult challenge for the designers. The most important parameters include initial bonding force $F$, number of particles $N$, the adhesion strength $G_{A}$, and modulus of elasticity $E$ of the cured resin. It is well known that as the bonding force increases, the contact resistance decreases. However, when the bonding force reaches a certain maximum value, the contact between conductive particle and conductive track is disrupted due to delamination of the cured resin during the elastic recovery. The authors have shown in previous studies that the delamination is caused by high residual stresses and that it largely depends on the adhesion strength of the assembly and on the modulus of elasticity of the cured resin. Additionally, the authors have provided a methodology to quantify the maximum threshold value of the bonding force for different numbers of particles trapped between mating conductive tracks. In this paper, the relationships between contact resistance $R$ and each one of these parameters are systematically investigated to create diagrams that give regions of robust design. Given the number of particles and their size, adhesion strength, and modulus of elasticity of the resin, the required bonding force can be found in order to achieve a desired range in contact resistance. [DOI: 10.1115/1.2912180]

Keywords: anisotropic conductive adhesive, bonding force, adhesion strength, electrical contact resistance, delamination

\section{Introduction}

Anisotropic conductive adhesive (ACA) assemblies are currently widely used in the flip chip technology [1]. The adhesive, or $\mathrm{ACA}$, is a composite material that generally consists of spherical conductive particles uniformly dispersed in an insulating resin matrix that possesses adhesive properties. The ACA is placed between chip and substrate to provide electrical and mechanical interconnection. Electrical conduction is achieved through the conductive particles trapped between the corresponding conductive tracks in chips and substrates when bonding force and heat are simultaneously applied. When the resin matrix is cured, the bonding force is removed. This adhesive matrix, usually a thermosetting epoxy resin, is expected to provide stable adhesion between the chip and substrate and electrical insulation where necessary. Schematics and pictures of ACA interconnects can be found in Ref. [2].

Although the ACA assembly method is widely used, there are concerns about their low conductivity for smaller scale applications. An accurate prediction of contact resistance in ACA joints has therefore become critically important. Among the parameters that influence this resistance, the bonding force used to compress the conductive particles against the conductive tracks during the assembly process is very important [3]. It is well known that as the bonding force increases, the contact resistance decreases [4-6]. However, when the bonding force reaches a certain maximum value, the contact between conductive particle and conduc-

\footnotetext{
Present address: Advanced Micro Devices, 1 AMD Place, Sunnyvale, CA 94085. ${ }^{2}$ Corresponding author.

Contributed by the Electrical and Electronic Packaging Division of ASME for publication in the Journal of Electronic Packaging Manuscript received January 17, 2006; final manuscript received September 9, 2007; published online May 8, 2008. Assoc. Editor: Mark D. Poliks. Paper presented at the 2005 ASME International Mechanical Engineering Congress (IMECE2005), Orlando, FL, November
} 5-11, 2005. tive track is disrupted due to delamination of the cured resin during the elastic recovery. It has been shown by the authors [7] that the delamination is caused by high residual stresses and that it largely depends on the modulus of elasticity and adhesion strength of the cured resin. Additionally, the authors have provided a methodology to quantify the maximum threshold value of the bonding force for different values of the number of particles trapped between mating conductive tracks [8].

This technical brief synthesizes the results of Refs. [7,8] into design guidelines on material and process parameter selection in the assembly of ACA interconnect. Specifically, the relationships between contact resistance $R$, initial bonding force $F$, modulus of elasticity $E$ of the resin, adhesion strength $G_{A}$ of the joint, and number of particles $N$ are combined to create diagrams that give regions of robust design. Sec. 2 describes the important parameters to be considered in the diagrams, Sec. 3 presents the methodology used to create the diagrams, and Secs. 4 and 5 give the results and conclusions.

\section{Analysis}

As reported in Ref. [7], the contact resistance changes as the bonding force is removed at the end of the assembly process (unloaded condition) when the epoxy resin used to bond the surfaces has been cured. It was concluded that $R$ highly depends on the values of $F, G_{A}$, and $E$. It was shown that smaller values of $G_{A}$ yield higher contact resistance, and similarly, smaller values of $E$ lead to higher contact resistance values [7]. The relationship between $R$ and $F$ was found to be different for the unloaded condition and the loaded condition. In the unloaded condition, it was found that delamination induces a sharp increase in contact resistance after a certain maximum threshold value of bonding force. In Ref. [8], the relationship between $R$ and $F$ for multiple particles 
in the unloaded condition was determined. It was found that as the number of particles increases, the maximum threshold value of $F$ that causes delamination increases.

From Refs. $[7,8]$, it can also be noticed that to accomplish contact, the initial bonding force $F$ needs to be larger than a certain minimum threshold value. Initially, the contact resistance sharply decreases until the bonding force reaches the minimum threshold value. As the bonding force keeps increasing, the contact resistance decreases at a slower pace up to a maximum threshold value of the bonding force. At this point, the contact resistance sharply increases because of the effect of elastic recovery, which is related to the modulus of elasticity and adhesion strength characterized in Ref. [7]. This maximum threshold value of bonding force also largely depends on the number of particles [8]. The larger the number of particles is, the larger the threshold value is. This behavior is due to the amount of residual stress in the deformed particles. The larger the number of particles is, the less the amount of residual stress in each of them is, and therefore, the delamination is less likely to occur [8].

Because of the behavior depicted above, it is important to select ACA materials and process parameters that allow the initial bonding force to be between the minimum and maximum threshold values of the specific ACA configuration to ensure reasonable values of contact resistance. The minimum threshold value is given by the required acceptable value of contact resistance and by the material properties of the conductive particles and tracks. This value can be found in a straightforward manner from traditional mechanics analysis. The maximum threshold value, on the other hand, depends on the number of particles, adhesive strength, and modulus of elasticity of the cured resin. The influence of each parameter is separately depicted in Refs. [7,8], but the interrelationship between them has not been defined. In the next section, a methodology to combine the information in Refs. [7,8] is developed for ACA design. The interrelationship between adhesion strength, modulus of elasticity, number of particles, and bonding force is found and used to find specific zones of robust design.

\section{Methodology}

The interrelationship between $F, G_{A}$, and $E$ is characterized by combining the relationships described in Fig. 11 in Ref. [7] (hereafter referred to as Graph 1) and Fig. 13 in Ref. [8] (hereafter referred to as Graph 2). Since these relationships both describe contact resistance, their values are used to associate the two graphs. From Graph 1, each value of contact resistance gives three sets of values of $E$ and $G_{A}$. Each contact resistance corresponds in turn to one specific value of $F$ in Graph 2. A curve $E$ versus $G_{A}$ for each value of $F$ is then traced. Each curve of $F$ marks the minimum allowable values of $E$ and $G_{A}$ for that specific value of $F$. The curves are traced as many times as required to depict the complete behavior shown in Graph 2.

The procedure described above is carried out for different numbers of particles $N$. Notice that since the curve in Fig. 2 is valid only for a single particle model, the values of contact resistance used to define the bonding force were multiplied by the corresponding factor of the number of particles.

Notice also that the same material properties and geometry assumed in Refs. $[7,8]$ are valid in this work as well. Specifically, the spherical particles are assumed to be solid metal made of nickel and of radius $r=5 \mu \mathrm{m}$. The conductive track is made of copper and of thickness $t=12 \mu \mathrm{m}$. The material properties of the nickel and copper considered are listed in Ref. [7]. The resin was assumed to be nonconductive and with a low viscosity at the beginning of the bonding process; when cured, it was assumed to be nonconductive and with Poisson's ratio $\nu=0.4$.

\section{Results and Discussion}

Figure 1 illustrates the relationships of $F, E$, and $G_{A}$ for a single particle case. Each of the curves of $F$ marks the minimum allow-

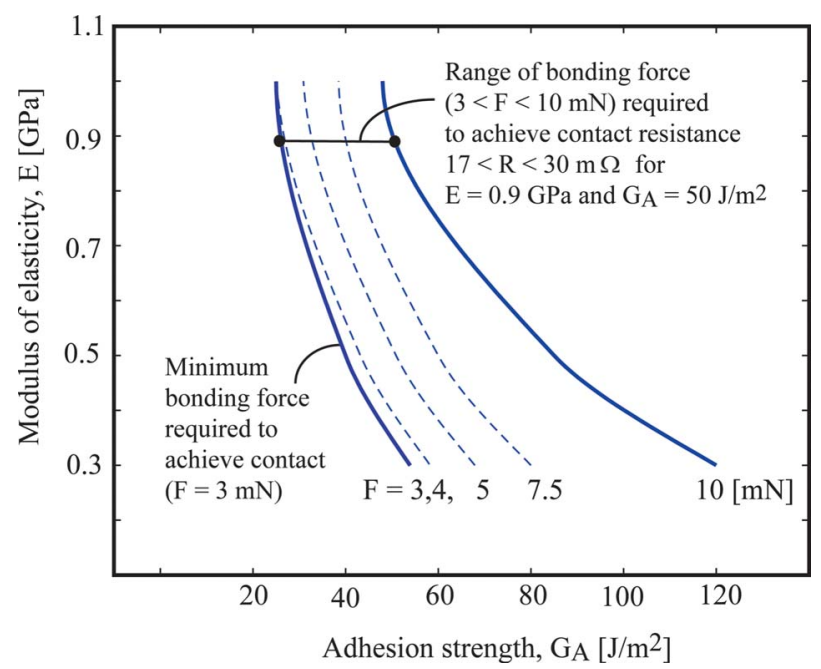

Fig. 1 Interrelationship between modulus of elasticity $E$, adhesion strength $G_{A}$, and initial bonding force $F$ for the single particle case

able values of $E$ and $G_{A}$ for that specific value of $F$. That is, any set of the $E$ and $G_{A}$ values found on the left side of the $F$ curve will cause delamination. Any set on the right side will maintain contact. A different interpretation of the graph is that for a given set of values of $E$ and $G_{A}$, the value of the $F$ curve that it intersects represents the maximum threshold value. For example, the values of $E=0.9 \mathrm{GPa}$ and $G_{A}=50 \mathrm{~J} / \mathrm{m}^{2}$ intersect at the curve $F$ $=10 \mathrm{mN}$. As shown in Graph 2, the value of $F=10 \mathrm{mN}$ represents the maximum threshold value of the single particle case for the same given set of $E$ and $G_{A}$ and that the corresponding resistance is $R=17 \mathrm{~m} \Omega$. It can also be noted that if $F=3 \mathrm{mN}$, the resistance is $R=30 \mathrm{~m} \Omega$. In other words, if the bonding force is within the range $3<F<10 \mathrm{mN}$, the resistance will be correspondingly within the range $17<R<30 \mathrm{~m} \Omega$. This is also shown in Fig. 1 but with all the combination of permissible values of $E$ and $G_{A}$ for $F=10 \mathrm{mN}$ and for different values of $F$ as well.

The interrelationship between $E, G_{A}$, and number of particles $N$ is shown in Fig. 2. The curves of $F=20 \mathrm{mN}$ for different numbers of particles are shown (solid lines). The graph reveals that as the number of particles increases, the limiting set of values of $E$ and

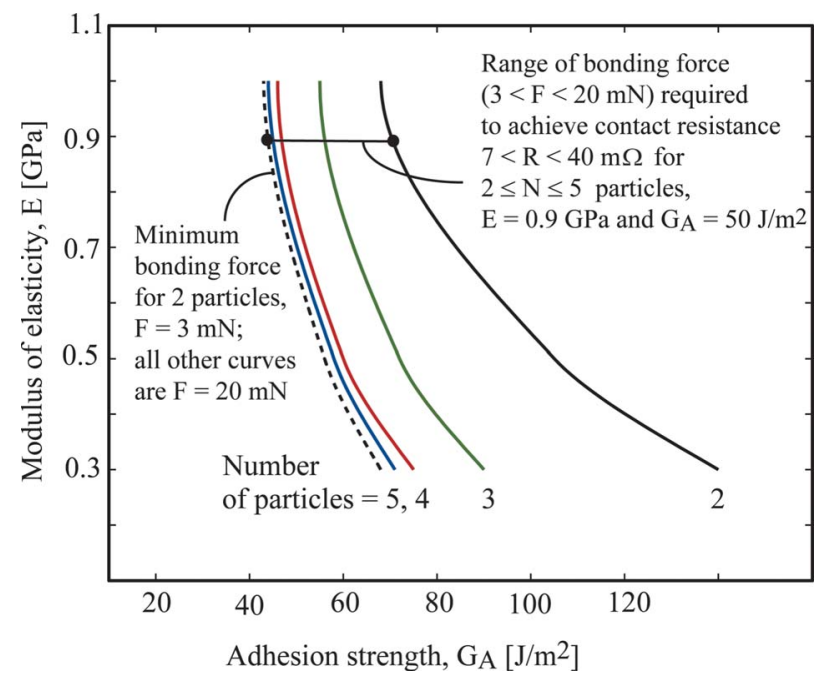

Fig. 2 Interrelationship between modulus of elasticity $E$, adhesion strength $G_{A}$, and number of particles $N$ for an initial bonding force of $20 \mathrm{mN}$ and multiple particles 
$G_{A}$ decreases (the curves shift toward the left). This is because the larger the number of particles, the smaller the amount of residual stress on each particle. The graph also shows that the specific values of $E=0.9 \mathrm{GPa}$ and $G_{A}=50 \mathrm{~J} / \mathrm{m}^{2}$ indicate a maximum threshold value of $F=20 \mathrm{mN}$ for the two-particle case. This is in accordance with Graph 2. Figure 2 also shows, however, that if there were three, four, or five particles instead of just two, contact is still maintained and that by including the curve of the minimum threshold value, $F=2.5 \mathrm{mN}$, of the two-particle arrangement (dotted line), it can be noticed that a range of bonding force $2.5<F$ $<20 \mathrm{mN}$ and of particles $2 \leq N \leq 5$ gives a range of contact resistance $7<R<40 \mathrm{~m} \Omega$.

A critical question when using these design guidelines is how many particles are expected in a practical situation. The number of particles trapped between conductive tracks depends on the volume fraction of the particles in the adhesive, the size of the particles, and the size of the conductive tracks. Useful mathematical and statistical methods to estimate the number of particles based on these parameters can be found in Ref. [9]. Alternatively, the diagrams can be reworked by including deformation of the particles instead of bonding force. In such case, the number of particles would not be relevant in the diagrams.

\section{Conclusions}

The independent relationships of contact resistance versus initial bonding force, number of particles, adhesion strength, and contact resistance versus modulus of elasticity of the cured resin have been combined to create design diagrams, which gives regions of robust electrical contact resistance. The diagrams, provide design guidelines to assemble, in a convenient manner, ACA interconnects free of delamination. By using these graphs, two cases of robust design were described: (1) determination of a preferred range of bonding force to achieve a certain contact resistance in a single particle ACA assembly with given values of $E$ and $G_{A}$ and (2) determination of a preferred range of bonding force to achieve a certain contact resistance in a multiparticle ACA assembly with given values of $E$ and $G_{A}$ and the number of particles.

\section{References}

[1] Lau, J., 1996, Flip Chip Technologies, McGraw-Hill, New York.

[2] Liu, J., 1999, Conductive Adhesives for Electronics Packaging, Electrochemical Publications Ltd., London, UK.

[3] Chin, M., Iyer, K., and Hu, S. J., 2004, "Prediction of Electrical Contact Resistance for Anisotropic Conductive Adhesive Assemblies," IEEE Trans. Compon. Packag. Technol., 27(2), pp. 317-326.

[4] Yim, M. J., and Paik, K. W., 1998, "Design and Understanding of Anisotropic Conductive Adhesive (ACF's) for LCD Packaging," IEEE Trans. Compon. Packag. Manuf. Technol., Part A, 21(2), pp. 226-234.

[5] Hu, K. X., Yeh, C. P., and Wyatt, K. W., 1997, "Electro-Thermo-Mechanical Responses of Conductive Adhesive Materials," IEEE Trans. Compon., Packag. Manuf. Technol., Part A, 20(4), pp. 470-477.

[6] Fu, Y., Willander, M., and Liu, J., 2001, "Statistics of Electric Conductance Through Anisotropically Conductive Adhesive," IEEE Trans. Compon. Packag. Technol., 24(2), pp. 250-255.

[7] Chin, M., Barber, J. R., and Hu, S. J., 2006, "Effect of Elastic Recovery on the Electrical Contact Resistance in Anisotropic Conductive Adhesive Assemblies," IEEE Trans. Compon. Packag. Technol., 29(1), pp. 137-144.

[8] Chin, M., and Hu, S. J., 2007“A Multiple Particle Model for the Prediction of Electrical Contact Resistance in Anisotropic Conductive Adhesive Assemblies," IEEE Trans. Compon., Packag. Manuf. Technol., Part A 30(4), 745753.

[9] Williams, D. J., and Whalley, D. C., 1993, "The Effects of Conducting Particle Distribution on the Behavior of Anisotropic Conductive Adhesives: NonUniform Conductivity and Shorting Between Connections," J. Electron. Manuf., 3, pp. 85-94. 